\title{
CURVATURE CONDITION FOR NON-CONTRACTIONS DOES NOT IMPLY SIMILARITY TO THE BACKWARD SHIFT.
}

\author{
HYUN-KYOUNG KWON AND SERGEI TREIL
}

\begin{abstract}
We give an example of an operator that satisfies the curvature condition as defined in [2, but is not similar to the backward shift $S^{*}$ on the Hardy class $H^{2}$. We conclude therefore that the contraction assumption in the similarity characterization given in 2] is a necessary requirement.
\end{abstract}

\section{Contents}

Notation

1. Introduction and result

2. The construction

3. Main estimates

References

:= $\quad$ equal by definition;

$\mathbb{C} \quad$ the complex plane;

$\mathbb{D} \quad$ the unit disk, $\mathbb{D}:=\{z \in \mathbb{C}:|z|<1\}$;

$\frac{\partial}{\partial z}, \frac{\partial}{\partial \bar{z}} \quad \partial$ and $\bar{\partial}$ derivatives: $\frac{\partial}{\partial z}:=\left(\frac{\partial}{\partial x}-i \frac{\partial}{\partial y}\right) / 2, \frac{\partial}{\partial \bar{z}}:=\left(\frac{\partial}{\partial x}+i \frac{\partial}{\partial y}\right) / 2$, the symbols $\partial$ and $\bar{\partial}$ are sometimes used;

$\Delta \quad$ normalized Laplacian, $\Delta=\bar{\partial} \partial=\partial \bar{\partial}=\frac{1}{4}\left(\frac{\partial^{2}}{\partial x^{2}}+\frac{\partial^{2}}{\partial y^{2}}\right) ;$

$\|\cdot\| \quad$ norm;

$H^{2}, H^{\infty} \quad$ Hardy classes of analytic functions,

$$
H^{p}:=\left\{f \in L^{p}(\mathbb{T}): \hat{f}(k):=\int_{\mathbb{T}} f(z) z^{-k} \frac{|d z|}{2 \pi}=0 \text { for } k<0\right\},
$$

2000 Mathematics Subject Classification. Primary 47A99, Secondary 47B32, 30D55, $53 \mathrm{C} 55$.

The work of S. Treil was supported by the National Science Foundation under Grant DMS-0501065. 
Hardy classes can be identified with the spaces of functions that are analytic in the unit disk $\mathbb{D}$ : in particular, $H^{\infty}$ is the space of all functions bounded and analytic in $\mathbb{D}$.

\section{INTRODUCTION AND RESULT}

In this paper we consider operators with a complete analytic family of eigenvectors (the backward shift in the Hardy space $H^{2}$, or, more generally in some reproducing kernel Hilbert space being a typical example). We are interested in the classification of such operators in terms of the geometry of their eigenvector bundles.

The problem of unitary classification was completely solved by M. J. Cowen and R. G. Douglas in [1]. One of the first results in [1] was a version of Calabi's Rigidity Theorem, which stated that if the eigenvector bundles of operators $T_{1}$ and $T_{2}$ are isomorphic (as Hermitian vector bundles), then the operators are unitarily equivalent. In the case of $\operatorname{dim} \operatorname{ker}\left(T_{1,2}-\lambda I\right)=1$, the fact that the eigenvector bundles are isomorphic simply means that the curvatures coincide. Note, that the general case of $\operatorname{dim} \operatorname{ker}\left(T_{1,2}-\lambda I\right)=$ $n<\infty$ was also treated in [1], and numerous local criteria for determining unitary equivalence were obtained there.

The problem of classification of operators up to similarity, as some examples in [1] did show, is significantly more complicated. For example, an obvious conjecture that uniform equivalence of curvatures is sufficient for similarity (it is obviously necessary) is trivially wrong; the curvatures of the (eigenvector bundles of the) backward shifts in $H^{2}$ and in the Bergman space $A^{2}$ differ by a factor of 2 , but these operators are very far from being similar.

In 2] a particular case of the similarity problem, namely the problem of similarity to the backward shift in the Hardy space $H^{2}$ was considered. The structure of the backward shift is very well understood, so there was a hope that it will be possible to get a solution in this special case, and that this solution may lead to a better understanding of the general case.

This program was partially realized by the authors: in [2] a complete description (in terms of the geometry of the eigenvector bundles) of operators similar to backward shifts of finite multiplicity was obtained, but under an additional assumption that the operator $T$ is a contraction, $\|T\| \leq 1$.

More precisely, it was assumed in 2 that the operator $T$ in a Hilbert space $H$ satisfies the following 4 conditions:

(i) $T$ is contractive, i.e., $\|T\| \leq 1$;

(ii) $\operatorname{dim} \operatorname{ker}(T-\lambda I)=n<\infty$ for all $\lambda \in \mathbb{D}$;

(iii) $\operatorname{span}\{\operatorname{ker}(T-\lambda I): \lambda \in \mathbb{D}\}=H$;

(iv) The subspaces $\mathcal{E}(\lambda)=\operatorname{ker}(T-\lambda I)$ depend analytically on the spectral parameter $\lambda \in \mathbb{D}$.

The main result of [2] was a necessary and sufficient condition for the operator $T$ to be similar to the backward shift of multiplicity $n$, i.e., to the 
direct sum of $n$ backward shifts $S^{*}$ in $H^{2}, S^{*} f(z)=(f(z)-f(0)) / z, f \in H^{2}$. For the case $n=1$, which we are considering in this paper, this result can be stated as follows:

Theorem 1.1 (Theorem 0.1, 2]). The following statements are equivalent:

(1) $T$ is similar to the backward shift operator $S^{*}$ on $H^{2}$;

(2) The eigenvector bundles of $T$ and $S^{*}$ are "uniformly equivalent", i.e., there exists a holomorphic bundle map bijection $\Psi$ from the eigenvector bundle of $S^{*}$ to that of $T$ such that for some constant $c>0$

$$
\frac{1}{c}\left\|v_{\lambda}\right\|_{H^{2}} \leq\left\|\Psi\left(v_{\lambda}\right)\right\|_{H} \leq c\left\|v_{\lambda}\right\|_{H^{2}}
$$

for all $v_{\lambda} \in \operatorname{ker}\left(S^{*}-\lambda I\right)$ and for all $\lambda \in \mathbb{D}$;

(3) There exists a bounded subharmonic solution $\varphi$ to the Poisson equation

$$
\Delta \varphi(z)=\left|\kappa_{S^{*}}(z)-\kappa_{T}(z)\right|
$$

for all $z \in \mathbb{D}$, where $\kappa_{S^{*}}$ and $\kappa_{T}$ denote the curvatures of the eigenvector bundles of the operators $S^{*}$ and $T$, respectively. We say that the "curvature condition" is satisfied in this case.

(4) The measure

$$
\left|\kappa_{S^{*}}(z)-\kappa_{T}(z)\right|(1-|z|) d x d y,
$$

where $\kappa_{S^{*}}$ and $\kappa_{T}$ are the corresponding curvatures, is Carleson and the estimate

$$
\left|\kappa_{S^{*}}(z)-\kappa_{T}(z)\right|^{\frac{1}{2}} \leq \frac{C}{1-|z|}
$$

holds.

Remark 1.2. Without stating the exact definition, let us recall that the curvature $\kappa_{T}$ of the eigenvector bundle of $T$ (in fact, the curvature of any Hermitian line bundle over a planar domain) can be calculated as $\kappa_{T}(z)=$ $-\Delta \ln \|\gamma(z)\|^{2}$, where $\gamma$ is a section of the bundle and $\Delta$ is the normalized Laplacian, i.e., $\Delta=\bar{\partial} \partial=\partial \bar{\partial}[1$.

It can also be expressed as $\kappa_{T}(z)=-\left|\frac{\partial \Pi(z)}{\partial z}\right|^{2}$, where $\Pi: \mathbb{D} \rightarrow B(H)$ denotes the projection-valued function that assigns to each $\lambda \in \mathbb{D}$, the orthogonal projection onto $\operatorname{ker}(T-\lambda I), \Pi(\lambda):=P_{\operatorname{ker}(T-\lambda I)}$ (this formula is in fact true for any line subbundle of the trivial bundle $\Omega \times H \rightarrow \Omega$, where $H$ is a Hilbert space and $\Omega \subset \mathbb{C}$ ).

In [2, the latter formula for the curvature was used, but it is well known and not hard to show that both formulas give the same result.

Remark 1.3. For the backward shift $S^{*}$, the curvature $\kappa_{S^{*}}$ can be easily computed: $\kappa_{S^{*}}(z)=\left(1-|z|^{2}\right)^{-2}$.

Remark 1.4. It was shown in [2] that if $T$ is a contraction (with $\operatorname{dim}(T-$ $\lambda I)=1$ for all $\lambda \in \mathbb{D}$ ), then $\kappa_{S^{*}}-\kappa_{T} \geq 0$. So in the statement of the 
main theorem (Theorem 0.1) in [2], the term $\kappa_{S^{*}}-\kappa_{T}$ was used instead of $\left|\kappa_{S^{*}}-\kappa_{T}\right|$. This nonnegativity is consistent with a more general result in [3], but we can no longer take it for granted when it comes to noncontractions.

A natural question is whether it is possible to get rid of the assumption that $T$ is contractive in Theorem 1.1. This question was partially answered in [2], where a noncontractive operator satisfying statement (2), but which is not similar to $S^{*}$ was constructed. That means the assumption $\|T\| \leq 1$ is needed to prove Theorem 1.1 in full generality.

However, there was hope, that maybe condition (3) and/or (4) is sufficient for similarity even without assuming that $T$ is a contraction.

In the present paper, we destroy this hope by giving an example of an operator that satisfies statements (3) and (4), but is not similar to $S^{*}$. This example is a slight modification of the one given in [2].

Now we state the main result:

Theorem 1.5. Given $\alpha>0$, there exists an operator $T$ that is "almost" a coisometry in the sense that $(1-\alpha)\|x\| \leq\left\|T^{*} x\right\| \leq(1+\alpha)\|x\|$, and such that it is not similar to $S^{*}$ on $\mathrm{H}^{2}$, but satisfies statements (2)-(4) of Theorem 0.1 .

\section{The CONSTRUCTION}

The operator $T$ will be constructed as the backward shift on the weighted Hardy class $H_{w}^{2}:=\left\{f=\sum_{n \geq 0} a_{n} z^{n}:\|f\|_{w}^{2}:=\sum_{n \geq 0}\left|a_{n}\right|^{2} w_{n}<\infty\right\}$, where $w=\left\{w_{n}\right\}_{0}^{\infty}, w_{n}>0$. Let us remind the reader that the backward shift in $H_{w}^{2}$ is the adjoint of the forward shift $f \mapsto z f$, and it can be easily shown that

$$
T\left(\sum_{n=0}^{\infty} a_{n} z^{n}\right)=\sum_{n=0}^{\infty} \frac{w_{n+1}}{w_{n}} a_{n+1} z^{n} .
$$

Let us also remind the reader that the eigenvector bundle of $T$ is the family of the reproducing kernel $k_{\lambda}^{w}$ of $H_{w}^{2}$,

$$
T k_{\lambda}^{w}=\bar{\lambda} k_{\lambda}^{w}, \quad \lambda \in \mathbb{D},
$$

and that the reproducing kernel $k_{\lambda}^{w}$ can be computed by the following formula:

$$
k_{\lambda}^{w}(z)=\sum_{k=0}^{\infty} \frac{\bar{\lambda}^{n} z^{n}}{w_{n}} .
$$

The weight sequence $w=\left\{w_{n}\right\}_{0}^{\infty}\left(w_{n}>0\right)$ will be of the form (see Fig. 11)

$$
\ln w_{n}= \begin{cases}2 j \ln (1+\alpha) & n=N_{k}+j, 0 \leq j \leq k, \\ 2 j \ln (1+\alpha) & n=N_{k}+2 k-j, 0 \leq j \leq k, \\ 0 & \text { otherwise, }\end{cases}
$$

where the numbers $N_{k}, k \in \mathbb{N}$, satisfying $N_{k}+2 k<N_{k+1}$ will be chosen later. 


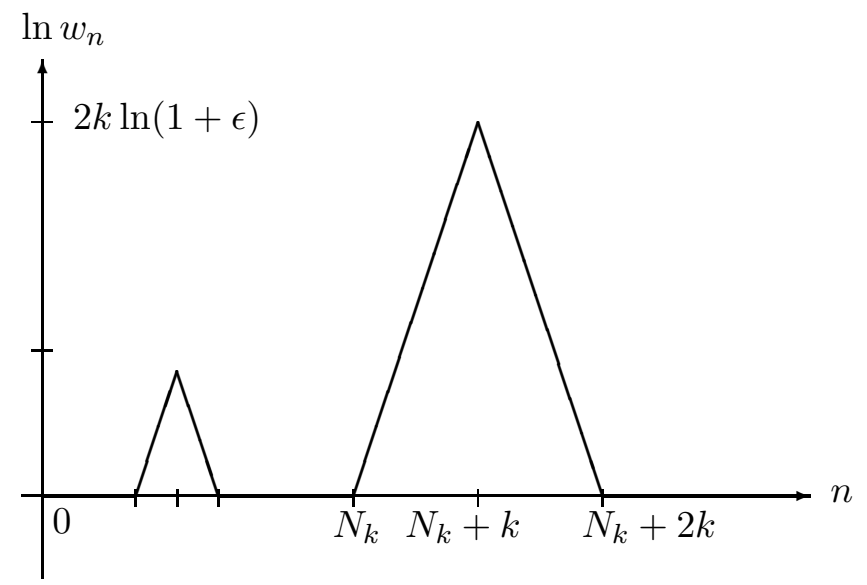

Figure 1. The function $\ln w_{n}$ : two "spikes" are shown

Using the fact that the weight sequence $w=\left\{w_{n}\right\}_{0}^{\infty}$ is unbounded, one can show that the operator $T$ is not similar to $S^{*}$. To see this, let us assume the contrary, i.e., $A T=S^{*} A$ for some bounded, invertible operator $A$ so that $T^{* n} A^{*}=A^{*} S^{n}$. If we take an $f \in H^{2}$ such that $A^{*} f=\sum_{0}^{\infty} a_{n} z^{n} \neq 0$ and an $m$ such that $a_{m} \neq 0$, then

$$
\left\|T^{* n} A^{*} f\right\|^{2}=\sum_{j=0}^{\infty}\left|a_{j}\right|^{2} w_{j+n} \geq\left|a_{m}\right|^{2} w_{m+n},
$$

so $\sup _{n}\left\|T^{* n} A^{*} f\right\|=\infty$. But $\left\|A^{*} S^{n} f\right\|_{H_{w}^{2}} \leq\left\|A^{*}\right\|\|f\|_{H^{2}}$, and we have a contradiction.

Also, it is easy to see that the adjoint $T^{*}$ of the operator is "almost" an isometry, i.e.,

$$
(1-\alpha)\|x\| \leq\left\|T^{*} x\right\| \leq(1+\alpha)\|x\|
$$

for all $x \in H_{w}^{2}$. This is due to the slope condition

$$
(1+\alpha)^{-2} \leq w_{n+1} / w_{n} \leq(1+\alpha)^{2},
$$

for all $n \geq 0$.

In the next section, we will prove that it is possible to choose the numbers $N_{k}$ so that statements (2)-(4) of Theorem 1.1 are true. Since statement (3) follows from (with estimates) (4) (see [2], [5]), it suffices to check only (2) and (4).

\section{Main estimates}

In fact, we will prove even more than what is stated in Theorem 1.5, Namely we will show that given $\varepsilon>0$, one can pick the numbers $N_{k}$ 's in such a way that 
(1) $(1+\varepsilon)^{-1} \leq\left|k_{\lambda}^{1}(\lambda) / k_{\lambda}^{2}(\lambda)\right| \leq 1+\varepsilon$ for all $\lambda \in \mathbb{D}$, where $k_{\lambda}^{1}$ and $k_{\lambda}^{2}$ are the reproducing kernels for the Hardy space $H^{2}$ and for the weighted Hardy space $H_{w}^{2}$, respectively 1 This means that condition (2) of Theorem 1.1 holds with $c=1+\varepsilon$ (the bundle map between the eigenvector bundles is $\left.k_{\bar{\lambda}}^{1} \mapsto k_{\bar{\lambda}}^{2}, \lambda \in \mathbb{D}\right)$.

(2) The difference of the curvatures satisfies the estimate

$$
\left|\kappa_{T}(z)-\kappa_{S^{*}}(z)\right| \leq \frac{\epsilon}{(1-|z|)^{2}}
$$

and the measure

$$
d \mu=\left|\kappa_{T}(z)-\kappa_{S^{*}}(z)\right|(1-|z|) d x d y
$$

is Carleson with Carleson norm $\|\mu\|_{\text {Carl }} \leq \epsilon$.

Let us first recall the definition of a Carleson measure. Denote by $I \subset \mathbb{T}$ an arc of the unit circle with length $|I|$. Then a positive measure $\mu$ in the closed unit disk is called a Carleson measure if

$$
\mu\left(Q_{I}\right) \leq c \frac{|I|}{2 \pi},
$$

for some constant $c>0$, where

$$
Q_{I}:=\left\{z \in \mathbb{C}: \frac{z}{|z|} \in I, 1-|z| \leq \frac{|I|}{2 \pi}\right\}
$$

is the Carleson window ([4] Chap. 6.3). The infimum of such $c>0$ is called the Carleson norm, denoted $\|\mu\|_{\text {Carl }}$.

The maps $z \mapsto k_{\bar{z}}^{1}$ and $z \mapsto k_{\bar{z}}^{2}$ are sections of the eigenvector bundles of the operators $S^{*}$ and $T$ respectively. Since $\left\|k_{\bar{z}}^{i}\right\|^{2}=\left(k_{\bar{z}}^{i}, k_{\bar{z}}^{i}\right)=k_{\bar{z}}^{i}(\bar{z})=$ $k_{z}^{i}(z)$, we get, recalling formula for the curvature (see Remark 1.2) that the corresponding curvatures equal $-\Delta \ln k_{z}^{i}(z), i=1,2$.

We know that $k_{z}^{1}(\lambda)=1 /(1-\bar{z} \lambda)$, so $k_{z}^{1}(z)=\frac{1}{1-|z|^{2}}$. On the other hand, (2.1) implies that

$$
k_{z}^{2}(z)=\sum_{n \geq 0} \frac{1}{w_{n}}|z|^{2 n}=\frac{1}{1-|z|^{2}}+\sum_{k=1}^{\infty} g_{k}(z),
$$

where

$$
g_{k}(z)=|z|^{2 N_{k}}\left(\sum_{j=1}^{k} c_{j}|z|^{2 j}+\sum_{j=1}^{k-1} c_{j}|z|^{2(2 k-j)}\right)
$$

where

$$
c_{j}=\frac{1}{(1+\epsilon)^{2 j}}-1
$$

\footnotetext{
${ }^{1}$ To shorten the notation we will use the symbol $k_{\lambda}^{2}$ instead of $k_{\lambda}^{w}$ for the reproducing kernel of $H_{w}^{2}$.
} 
Thus,

$$
\left|\kappa_{S^{*}}-\kappa_{T}\right|=\left|\Delta \ln \frac{k_{z}^{2}(z)}{k_{z}^{1}(z)}\right|=\left|\Delta \ln \left(1+\sum_{k=1}^{\infty} g_{k}(z)\left(1-|z|^{2}\right)\right)\right| .
$$

Since

$$
|\Delta \ln f|=\frac{\left.|f \Delta f-| \partial f\right|^{2} \mid}{|f|^{2}}
$$

to prove that conditions (1) and (2) hold, we need to show that given $\delta>0$, the $N_{k}$ 's can be chosen so that the following conditions hold for $f(z)=$ $\frac{k_{z}^{2}(z)}{k_{z}^{1}(z)}=1+\sum_{k=1}^{\infty} g_{k}(z)\left(1-|z|^{2}\right)$ :

(1) $1-\delta \leq|f| \leq 1+\delta$;

(2) (a) $|\Delta f| \leq \frac{\delta}{(1-|z|)^{2}}$;

(b) $|\partial f| \leq \frac{\sqrt{\delta}}{1-|z|}$;

(3) $d \mu=|\Delta f|(1-|z|) d x d y$ is a Carleson measure with $\|\mu\|_{\text {Carl }} \leq \delta$;

(4) $d \mu=|\partial f|^{2}(1-|z|) d x d y$ is a Carleson measure with $\|\mu\|_{C a r l} \leq \delta$.

Remark 3.1. Since $f$ is a quotient of the reproducing kernels, the above condition (1) implies condition (2) of Theorem 1.1 with $c=1 /(1-\delta)$.

But note that these conditions follow from the following four conditions where we consider the functions $h_{k}(z):=g_{k}(z)\left(1-|z|^{2}\right)$ instead of $f(z)$ :

(1) $\left|h_{k}\right| \leq \frac{\delta}{2^{k}}$

(2) (a) $\left|\Delta h_{k}\right| \leq \frac{\delta}{2^{k}(1-|z|)^{2}}$;

(b) $\left|\partial h_{k}\right| \leq \frac{\delta}{2^{k}(1-|z|)}$;

(3) $d \mu=\left|\Delta h_{k}\right|(1-|z|) d x d y$ is a Carleson measure with $\|\mu\|_{\text {Carl }} \leq \frac{\delta}{2^{k}}$;

(4) $d \mu=\left|\partial h_{k}\right|^{2}(1-|z|) d x d y$ is a Carleson measure with $\|\mu\|_{C a r l} \leq \frac{\delta}{2^{2 k}}$.

It is easy to see that the first three conditions for $h_{k}$ imply those for $f$ because they are just linear (in $h_{k}$ ) estimates. As for the fourth condition, let us notice that condition (4) for $h_{k}$ simply means that for every Carleson window $Q_{I}$,

$$
\left\|\partial h_{k}\right\|_{L^{2}\left(Q_{I},(1-|z|) d x d y\right)} \leq \frac{\sqrt{\delta}}{2^{k}}|I|^{\frac{1}{2}} .
$$

Since $\partial f=\sum_{k \geq 1} \partial h_{k}$, we conclude that

$$
\|\partial f\|_{L^{2}\left(Q_{I},(1-|z|) d x d y\right)} \leq \sum_{k=1}^{\infty} \frac{\sqrt{\delta}}{2^{k}}|I|^{1 / 2}=\sqrt{\delta}|I|^{1 / 2},
$$

which is exactly statement (4) for $f$.

Next, note that since the $h_{k}$ can be written down as $h_{k}=z^{2 N_{k}}(1-$ $\left.|z|^{2}\right) \sum_{j=1}^{2 k-1} a_{j}^{k}|z|^{2 j}$, it suffices to show that for the elementary functions

$$
\psi_{N}=|z|^{2 N}\left(1-|z|^{2}\right)=z^{N} \bar{z}^{N}-z^{N+1} \bar{z}^{N+1},
$$


the analogues of the above conditions (1)-(4) hold, and that by taking sufficiently large $N$ we can make the right sides of the inequalities there as small as we want. Hence Theorem 1.5 will follow from the Lemma given below:

Lemma 3.2. The following statements are true for the function $\psi_{N}=$ $|z|^{2 N}\left(1-|z|^{2}\right)$ :

(1) $\psi_{N}(z) \longrightarrow 0$ uniformly on $\mathbb{D}$ as $N \rightarrow \infty$;

(2) $\left|\Delta \psi_{N}(z)\right|(1-|z|)^{2} \longrightarrow 0$ uniformly on $\mathbb{D}$ as $N \rightarrow \infty$;

(3) $\left|\frac{\partial \psi_{N}(z)}{\partial z}\right|^{2}(1-|z|)^{2} \longrightarrow 0$ uniformly on $\mathbb{D}$ as $N \rightarrow \infty$;

(4) The measure $d \mu=d \mu_{N}=\left|\Delta \psi_{N}(z)\right|(1-|z|) d x d y$ is a Carleson measure with $\|\mu\|_{\text {Carl }} \rightarrow 0$ as $N \rightarrow \infty$;

(5) The measure $d \mu=d \mu_{N}=\left|\frac{\partial \psi_{N}(z)}{\partial z}\right|^{2}(1-|z|) d x d y$ is a Carelson measure with $\|\mu\|_{\text {Carl }} \rightarrow 0$ as $N \rightarrow \infty$.

Proof. Direct calculations show that

$$
\begin{aligned}
& \frac{\partial \psi_{N}}{\partial z}=N z^{N-1} \bar{z}^{N}-(N+1) z^{N} \bar{z}^{N+1}, \text { and } \\
& \frac{\partial \psi_{N}}{\partial \bar{z}}=N z^{N} \bar{z}^{N-1}-(N+1) z^{N+1} \bar{z}^{N} .
\end{aligned}
$$

We then have that

$$
\begin{aligned}
\Delta \psi_{N} & =N^{2}|z|^{2(N-1)}-(N+1)^{2}|z|^{2 N}, \text { and } \\
\left|\frac{\partial \psi_{N}}{\partial z}\right|^{2} & =|z|^{2(2 N-1)}\left(N-(N+1)|z|^{2}\right)^{2} .
\end{aligned}
$$

Since the maximum of $\psi_{N}$ attained at $|z|=\sqrt{\frac{N}{N+1}}$ is $\left(\frac{N}{N+1}\right)^{N} \frac{1}{N+1}$, statement (1) is obvious.

Let us prove (2). Denoting $r=|z|$, we have

$$
\begin{aligned}
\left|\Delta \psi_{N}(z)\right|(1-|z|)^{2} & =\left|N^{2} r^{2 N-2}-(N+1)^{2} r^{2 N}\right|(1-r)^{2} \\
& =\left|(N+1)^{2} r^{2 N-2}\left(1-r^{2}\right)-(2 N+1) r^{2 N-2}\right|(1-r)^{2} \\
& \leq 2(N+1)^{2} r^{2 N-2}(1-r)^{3}+(2 N+1) r^{2 N-2}(1-r)^{2} .
\end{aligned}
$$

We can see that both terms on the right side of the inequality can be estimated by functions of the same type:

$$
\begin{array}{ll}
(N+1)^{2} r^{2 N-2}(1-r)^{3} \leq C\left(n^{k} r^{k n}(1-r)\right)^{3}, & k=2 / 3, n=N-1, \\
(2 N+1) r^{2 N-2}(1-r)^{2} \leq C\left(n^{k} r^{k n}(1-r)\right)^{2}, & k=1 / 2, n=2(N-1) .
\end{array}
$$

The function $f(r)=n^{k} r^{k n}(1-r)$ attains its maximum value

$$
\left(\frac{k n}{k n+1}\right)^{k n} \frac{n^{k}}{k n+1}
$$


at $r=k n /(k n+1)$. Since $k<1$, the maximum value clearly goes to 0 as $n \rightarrow \infty$.

Estimating the square root of the expression in statement (3), we get

$$
\begin{aligned}
\left|\frac{\partial \psi_{N}(z)}{\partial z}\right|(1-|z|) & =r^{2 N-1}\left|N-(N+1) r^{2}\right|(1-r) \\
& \leq r^{2 N-1}\left|(N+1)\left(1-r^{2}\right)\right|(1-r)+r^{2 N-1}(1-r) \\
& \leq 2 r^{2 N-1}(N+1)(1-r)^{2}+r^{2 N-1}(1-r) .
\end{aligned}
$$

Since the maxima for the two terms on the right side of the last inequality are

$$
2(N+1)\left(\frac{2 N-1}{2 N+1}\right)^{2 N-1}\left(\frac{2}{2 N+1}\right)^{2}
$$

and

$$
\left(1-\frac{1}{2 N}\right)^{2 N-1} \frac{1}{2 N},
$$

respectively, we see that $\left|\frac{\partial \psi_{N}(z)}{\partial z}\right|(1-|z|)$ approaches 0 as $N \rightarrow \infty$.

For statement (4), let $S_{I}=\left\{z \in \mathbb{D}: \frac{z}{|z|} \in I\right\}$ be the smallest sector of $\mathbb{D}$ with vertex at 0 containing $Q_{I} \cap \mathbb{D}$. Since

$$
\begin{aligned}
\frac{1}{|I|} \mu\left(Q_{I}\right) & \leq \frac{1}{|I|} \mu\left(S_{I}\right) \\
& =\frac{1}{|I|} \int_{S_{I}}\left(N^{2}|z|^{2(N-1)}-(N+1)^{2}|z|^{2 N}\right) d x d y,
\end{aligned}
$$

we get the following by integrating in polar coordinates:

$$
\begin{aligned}
\frac{1}{|I|} \mu\left(Q_{I}\right) & \leq \frac{1}{|I|}|I| \int_{0}^{1}\left(N^{2} r^{2(N-1)}-(N+1)^{2} r^{2 N}\right) r d r \\
& =\int_{0}^{1}\left|N^{2}-(N+1)^{2} r^{2}\right| r^{2 N-1}(1-r) d r \\
& \leq 2(N+1)^{2} \int_{0}^{1} r^{2 N-1}(1-r)^{2} d r+(2 N+1) \int_{0}^{1} r^{2 N-1}(1-r) d r \\
& =2(N+1)^{2}\left(\frac{1}{2 N}-\frac{2}{2 N+1}+\frac{1}{2 N+2}\right) \\
& \quad+(2 N+1)\left(\frac{1}{2 N}-\frac{1}{2 N+1}\right) \\
& =2(N+1)^{2} \mathrm{O}\left(\frac{1}{N^{3}}\right)+2(N+1) \mathrm{O}\left(\frac{1}{N^{2}}\right) .
\end{aligned}
$$

Finally, as with statement (4), replacing $Q_{I}$ with the sector $S_{I}$ and integrating in polar coordinates, we reduce statement (5) to the following 
estimate:

$$
\begin{aligned}
\int_{0}^{1} & r^{4 N-1}\left(N-(N+1) r^{2}\right)^{2}(1-r) d r \\
& \leq 8 \int_{0}^{1} r^{4 N-1}(N+1)^{2}(1-r)^{3} d r+2 \int_{0}^{1} r^{4 N-1}(1-r) d r \\
& =8(N+1)^{2}\left(\frac{1}{4 N}-\frac{3}{4 N+1}+\frac{3}{4 N+2}-\frac{1}{4 N+3}\right)+2\left(\frac{1}{4 N}-\frac{1}{4 N+1}\right) \\
& =8(N+1)^{2} \mathrm{O}\left(\frac{1}{N^{4}}\right)+2 \mathrm{O}\left(\frac{1}{N^{2}}\right) .
\end{aligned}
$$

The lemma follows by letting $N \rightarrow \infty$.

\section{REFERENCES}

[1] M. J. Cowen and R. G. Douglas, Complex geometry and operator theory, Acta. Math., 141 (1978), 187-261.

[2] H. Kwon and S. Treil, Similarity of operators and geometry of eigenvector bundles, Publicacions Matematiques, to appear.

[3] G. Misra, Curvature inequalities and extremal properties of bundle shifts, J. Oper. Theory., 11 (1984), 305-317.

[4] N. K. Nikolski, Operators, Functions, and Systems: An Easy Reading. Vol. 1: Hardy, Hankel, and Toeplitz, Mathematical Surveys and Monographs, vol. 92, American Mathematical Society, Providence, RI, 2002, Translated from the French by Andreas Hartmann.

[5] _ Treatise on the Shift Operator, Grundlehren der Mathematischen Wissenschaften [Fundamental Principles of Mathematical Sciences], vol. 273, SpringerVerlag, Berlin, 1986, Spectral function theory, With an appendix by S. V. Hruščev [S. V. Khrushchëv] and V. V. Peller, Translated from the Russian by Jaak Peetre.

Department of Mathematics, Brown University, 151 Thayer Street Box 1917, Providence, RI USA 02912 